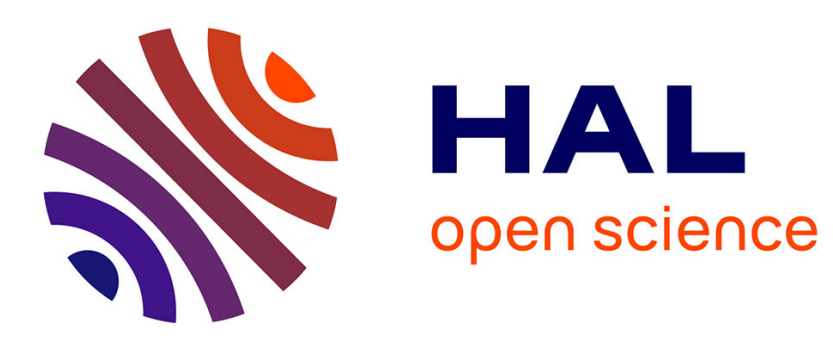

\title{
Application of metamodel-based techniques for the efficient seismic analysis of structural systems
}

Elisa Ferrario, Nicola Pedroni, Enrico Zio, Fernando Lopez-Caballero

\section{To cite this version:}

Elisa Ferrario, Nicola Pedroni, Enrico Zio, Fernando Lopez-Caballero. Application of metamodelbased techniques for the efficient seismic analysis of structural systems. ESREL 2015, Sep 2015, Zurich, Switzerland. pp.1193-1200. hal-01270627

\section{HAL Id: hal-01270627 \\ https://hal.science/hal-01270627}

Submitted on 8 Feb 2016

HAL is a multi-disciplinary open access archive for the deposit and dissemination of scientific research documents, whether they are published or not. The documents may come from teaching and research institutions in France or abroad, or from public or private research centers.
L'archive ouverte pluridisciplinaire HAL, est destinée au dépôt et à la diffusion de documents scientifiques de niveau recherche, publiés ou non, émanant des établissements d'enseignement et de recherche français ou étrangers, des laboratoires publics ou privés. 


\title{
Application of metamodel-based techniques for the efficient seismic analysis of structural systems
}

\author{
E. Ferrario \& N. Pedroni \\ Chair on Systems Science and the Energetic Challenge, Fondation EDF, at CentraleSupélec, France
}

E. Zio

Chair on Systems Science and the Energetic Challenge, Fondation EDF, at CentraleSupélec, France

Department of Energy, Politecnico di Milano, Italy

F. Lopez-Caballero

Laboratory MSSMat, UMR CNRS 8579, CentraleSupélec, France

\begin{abstract}
We look at the (failure) behavior of structural systems under the occurrence of seismic events. Detailed, long-running Finite Element Models (FEMs) are typically adopted for the simulation of the system structural response. However, since a very large number of simulations (e.g., several thousands) is usually required for an accurate assessment of the system failure behavior under different seismic conditions, the computational burden associated to the approach may be at times impractical. To address this issue, we resort to (fast-running) Artificial Neural Network (ANN) regression models, optimally trained to approximate the response of the original FEM. In particular, we embrace a wrapper approach based on Genetic Algorithm (GA) optimization to search for the optimal set of model inputs (features) that maximize the ANN representation accuracy. The ANN is trained, validated and tested with respect to the optimal feature subset identified; then, the results are compared with those produced by the original FEM. We illustrate the approach on a case study of seismic risk assessment involving the estimation of the fragility curves for a masonry structure.
\end{abstract}

\section{INTRODUCTION}

The Fukushima nuclear accident has highlighted the need for further research into nuclear safety and radiation protection. For this purpose, in 2013 a 5-year project called SINAPS@ (Earthquake and Nuclear Facilities: Ensuring Safety and Sustaining) has been launched in France. One of the key aspects of the project is the quantitative assessment of the (failure) behavior of Nuclear Power Plants (NPPs) under the occurrence of a seismic event. With respect to this objective, in this paper we preliminary study the failure behavior of a structural system subject to seismic risk; in particular, we identify the structure fragility curves representing the conditional probability of failure of a component for any given ground motion level (EPRI 2003).

In general, within this framework of analysis, the actions, events and physical phenomena that may cause damages to a nuclear (structural) system, are described by complex mathematical models, which are then implemented into computer codes to simulate the behavior of the system of interest under various conditions (USNRC 2009, NASA 2010). In particular, computer codes based on Finite Element Models (FEMs) are typically adopted for the simulation of the system structural behavior and response: an example is represented by Gefdyn code (Aubry et al. 1986).

In practice, not all the system characteristics can be fully captured in the mathematical model. This is due to the fact that (i) many of the events and physical phenomena of interest are random in nature (e.g., the earthquake) and (ii) the knowledge of the analyst about the system and the phenomena involved is typically not exhaustive (e.g., the power level in the nuclear reactor). As a consequence, uncertainty is always present in the values of the input parameters and variables of the mathematical model.

In this light, two issues need to be considered: first, an accurate assessment of the system failure behavior typically requires a very large number (e.g., several thousands) of FEM simulations under many different scenarios and conditions to fully explore the wide range of uncertainties affecting the system; second, FEMs are computationally expensive and may require hours or even days to carry out a single simulation. This makes the computational burden associated to the analysis at times impracticable.

In this context, fast-running regression models, also called metamodels (such as Artificial Neural Networks (ANN), Local Gaussian Processes (LGP), Quadratic Response Surfaces (QRS), etc.), can be built by means of input-output data examples to approximate the response of the original long-running FEMs and used into the seismic analysis at their place. Since the metamodel response is obtained quickly, the problem of high computational times is circumvented. 
On the other hand, any algorithm involving the construction of a metamodel suffers when the dimensionality of the input parameter (i.e., feature) space increases, because the set of input-output data examples available becomes sparser with a power law relationship (Hemez \& Atamturktur 2011). This issue is unavoidable and limits the application of any algorithm belonging to the metamodel family to engineering problems with a small number of input parameters (say ten), unless some dimensionality reduction strategy is adopted (e.g., principal component analysis or feature selection). In addition, it has been shown experimentally that irrelevant and noisy features unnecessarily increase the complexity of the problem and can degrade modeling performance ( $\mathrm{Na}$ 1997, Emmanouilidis et al. 1999, Buckner et al. 2002, Verikas \& Bacauskiene 2002). Finally, reducing the number of features decreases the cost and time of collecting unnecessary data.

For these reasons, it is recommended to identify a (possibly optimal) subset of the input model parameters that are relevant and essential for the accurate quantification of the output of interest (Zio et al. 2006).

In this work, we explore the representation capabilities of ANN metamodels to approximate the response of a detailed FEM and we embrace a wrapper approach based on Genetic Algorithm (GA) optimization to search for the optimal set of model inputs (features) that maximize the ANN representation accuracy (Zio et al. 2006).

As the focus of the paper is the performance of the ANN, we consider a simple structure for our analyses. The approach is applied to the quantification of the structural damages of a masonry structure subject to a seismic event. In particular, we compute the maximal displacement and we estimate the corresponding fragility curve for a given damage threshold (Lopez-Caballero et al. 2011).

The remainder of the paper is organized as follows. In Section 2, the steps necessary to build the fragility curves are illustrated; in Section 3, a synthetic description of ANN metamodels is provided; in Section 4, the GA-based wrapper approach for feature selection is described; in Section 5, the case study and the main results of the analysis are presented; in Section 6, conclusions and future developments are provided.

\section{FRAGILITY CURVE ESTIMATION FOR THE ANALYSIS OF THE FAILURE BEHAVIOR OF STRUCTURAL SYSTEMS}

Within the framework of Seismic Probabilistic Risk Assessment (SPRA), a fragility curve, $F$, represents in probabilistic terms the seismic capacity of a component/structure. Actually, it is the conditional prob- ability of failure (i.e., of exceeding a level of damage) of a component/structure for any given ground motion level, $y$ (EPRI 2003). It is standard practice to model the seismic capacity by a lognormal probability distribution with parameters $\alpha$ and $\beta$, where $\alpha$ is the median ground motion intensity measure (IM) and $\beta$ the logarithmic standard deviation (EPRI 2003). Typically, these parameters are evaluated for each structure and component for critical failure modes by the Fragility Analysis (EPRI 2003). Then, the fragility curve is defined as (EPRI 2003):

$$
F(y)=\Phi\left[\frac{1}{\beta} \log \left(\frac{y}{\alpha}\right)\right]
$$

where $\Phi[\cdot]$ is the standard Gaussian cumulative distribution of the term in brackets.

In this paper, we construct the fragility curve of the structure by estimating the parameters $\alpha$ and $\beta$ through the following three main steps:

(a) structural system modeling;

(b) structural system behavior simulation;

(c) fragility curves estimation.

In more details, in step (a) a mathematical model of the system is built to quantify its performance indicator. A quantitative model for seismic risk analysis may be viewed as composed of three main elements: a vector $\boldsymbol{Y}=\left\{y_{1}, y_{2}, \ldots, y_{\mathrm{M}}\right\}$ containing all the uncertain input variables (e.g., the ground motion IM); a computer code to simulate the behavior of the system of interest; and an output vector $\mathbf{Z}=$ $\left\{z_{1}, z_{2}, \ldots\right\}$ describing the system response (e.g., the structural top displacement).

In step (b) the mathematical model is implemented in a computer code and used to simulate system behavior under different uncertain operational and accidental conditions. For this reason, a very large number (e.g., several thousands) of simulations is typically needed.

Traditionally, computer codes based on Finite Element Models (FEMs) are adopted for the simulation of structural systems behavior and response. Since FEMs are computationally expensive (e.g., they may require hours or even days to carry out a single simulation), the computational cost associated to the analysis may be prohibitive.

One possibility to overcome this computational issue is to resort to fast-running regression models (metamodels) instead of the detailed, long-running FEMs. In this work, we adopt Artificial Neural Networks (ANNs) optimally trained to reproduce the nonlinear relation between a vector $\boldsymbol{Y}$ of $M$ inputs (representing different characteristics of the seismic event, like peak ground velocity ( $p g v)$, Arias Intensity $\left(I_{\text {Arias }}\right)$, Spectral Intensity $(S I)$, etc.) and one output, that is the maximal structural top displacement $\delta$, i.e., $\mathbf{Z}=z_{1}=\delta$ (see Section 3 ).

Finally, using the data generated at step (b) above, in step (c) the fragility curve $F(y)$ is obtained 
for a given damage threshold $\delta^{*}$ of interest by estimating the parameters $\alpha$ and $\beta$ through the maximum likelihood method (Saez et al. 2011).

\section{ARTIFICIAL NEURAL NETWORKS}

In extreme synthesis, ANNs are computing devices inspired by the function of the nerve cells in the brain (Bishop 1995). They are composed of many parallel computing units (called neurons or nodes) arranged in different layers and interconnected by weighed connections (called synapses). Each of these computing units performs a few simple operations and communicates the results to its neighbouring units. From a mathematical viewpoint, ANNs consist of a set of nonlinear (e.g., sigmoidal) basis functions with adaptable parameters $\boldsymbol{w}$ that are adjusted by a process of training (on many different input/output data examples), i.e., an iterative process of regression error minimization (Rumelhart et al. 1986). ANNs have been demonstrated to be universal approximants of continuous nonlinear functions (under mild mathematical conditions) (Cybenko 1989), i.e., in principle, an ANN model with a properly selected architecture can be a consistent estimator of any continuous nonlinear function. Further details about ANN regression models are not reported here for brevity; the interested reader may refer to the cited references and the copious literature in the field. The particular type of ANN considered in this paper is the classical feed-forward ANN composed of three layers (input, hidden and output, see Figure 1) and trained by the error backpropagation algorithm.

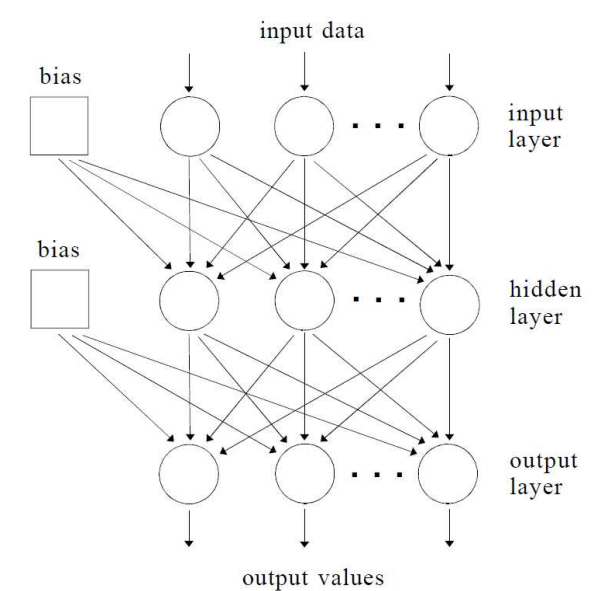

Figure 1. Scheme of a three-layered feed-forward Artificial Neural Network.

The number of nodes in the input layer equals the number of input variables $\boldsymbol{Y}$ which significantly affect the output; the number of nodes in the output layer is determined by the number of quantities $\boldsymbol{Z}$ of interest to the problem; and the number of nodes in the hidden layer in general is kept as low as possible, since the higher the number of hidden nodes the higher the number of parameters $(\boldsymbol{w})$ to be estimated. In general, an ANN with too few hidden nodes does not succeed in learning the training data set; vice versa, an ANN with too many hidden nodes learns the training data set very well, but it does not have generalization capability.

Typically, the entire set of input-output data is divided into three subsets:

- a training (input/output) data set, adopted to build the network, i.e., to calibrate the adjustable parameters $(\boldsymbol{w})$ of the regression model, for best fitting the FEM data;

- a validation (input/output) data set (made of patterns different from those of the training set), used to monitor the accuracy of the ANN model during the training procedure. In practice, the validation error is computed on the validation set at different iterative stages of the training procedure: at the beginning of training, this value decreases as does the error computed on the training set; later in the training, if the ANN regression model starts overfitting the data, the error calculated on the validation set starts increasing and training process must be stopped (Bishop, 1995);

- a test (input/output) data set, not used during ANN training and validation, needed to evaluate the network generalization capability in the presence of new data.

\section{GENETIC ALGORITHM-BASED WRAPPER FEATURE SELECTION FOR EFFICIENT ARTIFICIAL NEURAL NETWORK TRAINING AND CONSTRUCTION}

Any algorithm involving the construction of a metamodel suffers when the dimensionality of the input parameter (i.e., feature) space increases, because the set of input-output data examples available becomes sparser with a power law relationship (Hemez \& Atamturktur 2011). This issue is unavoidable and limits the application of any algorithm belonging to the metamodel family to engineering problems with a small number of input parameters, unless some dimensionality reduction strategy is adopted (e.g., principal component analysis or feature selection). In addition, it has been shown experimentally that irrelevant and noisy features unnecessarily increase the complexity of the problem and can degrade modeling performance ( $\mathrm{Na}$ 1997, Emmanouilidis et al. 1999, Buckner et al. 2002, Verikas \& Bacauskiene 2002). Finally, reducing the number of features decreases the cost and time of collecting unnecessary data. For these reasons, the optimal identification of a subset of $m$ features out of the $M$ originally available $(m \leq M)$ may increase the interpolation capabilities of the metamodel (Zio et al. 2006). 
The problem of feature selection is here formulated as an optimization problem. In this work, we select the important features within a wrapper approach where the feature selector behaves as a wrapper around the specific training algorithm used to construct the regression model. In this paper, the feature selector is represented by a Genetic Algorithm (GA) and the metamodel is an Artificial Neural Network (ANN) trained by error-back propagation.

The feature subsets are compared using as criterion the performance achieved by the ANN, i.e., the Root Mean Square Error (RMSE) on the test data set; obviously, the objective of the GA search is to find the feature subset for which the accuracy of the ANN is maximized, i.e., the RMSE is minimized. The inclusion or not of a feature in the subset can be encoded in terms of a binary variable which takes value 1 or 0 , respectively. For $M$ features, the size of the binary vector search space is $2^{M}$, thus an exhaustive search is impractical unless $M$ is small.

Let us define the total number of $M$-dimensional training and validation (input-output) data as the set $A$, the binary vector of dimension $M$ as the transformation vector $\boldsymbol{V}$, and the number of 1's in $\boldsymbol{V}$ as the number $m$ of selected features $(m \leq M)$. The GA creates a population of competing transformation vectors $\boldsymbol{V}_{i}, i=1,2, \ldots$, which are evaluated by the following steps (see Figure 2):

(a) The vector $V_{i}$ is applied to each pattern of the set $A$ to obtain a subset $B$ that represents the total number of $m$-dimensional data.

(b) The set $B$ of reduced features is the new input to the ANN. The network is trained, validated and tested. The RMSE on the test data set is computed.

The procedure is repeated until the minimum value of the RMSE on the test data set is found by the GA.

Notice that the GA searches also for the optimal number of hidden neurons, $h$, since it influences the performance of the ANN (see Section 3).

\section{FEATURE SELECTOR}

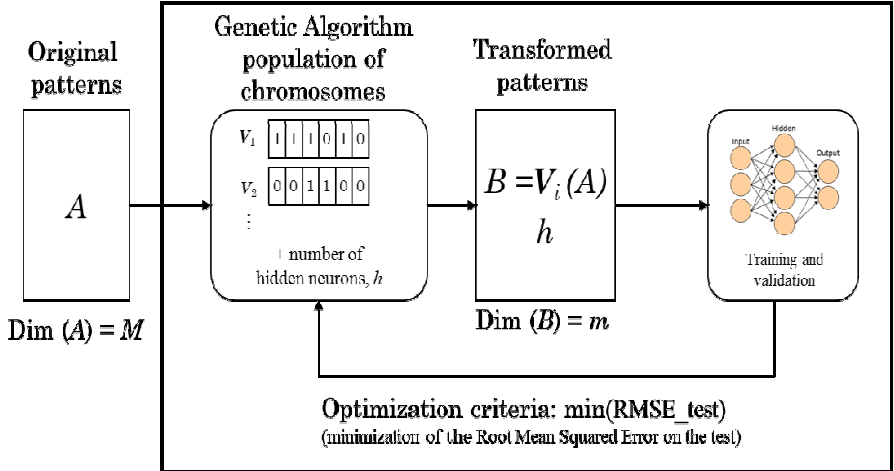

Figure 2. Structure of the Genetic Algorithm and Artificial Neural Network based feature selection.

\section{CASE STUDY}

We consider the non-linear soil behavior on the seismic response of a two-story masonry structures founded on a rigid shallow foundation.

In Section 5.1, the description of the specific system studied is given; in Section 5.2, the results of its evaluation are provided, together with some critical considerations.

\subsection{Case study description}

Figure 3 shows the masonry building analyzed in this work. The total height of the building is $5.4 \mathrm{~m}$, the width is $5.0 \mathrm{~m}$ and the thickness is $0.16 \mathrm{~m}$. With these characteristics the fundamental period of the structure $(T s t r)$ is equal to $0.19 \mathrm{~s}$. This structure is modelled using three different kinds of elements, beam-columns and diagonal struts describing the structural behavior and strengthless solid elements to represent the masonry mass. The frame's structural elements are modelled by plastic hinge beamcolumn elements. The behavior of this structure is simulated on the basis of non-linear dynamic Finite Element (FE) analysis. Further details about the masonry characteristics and the Finite Element Model (FEM) are not reported here for brevity sake, the interested reader is referred to (Lopez-Caballero et al. 2011).

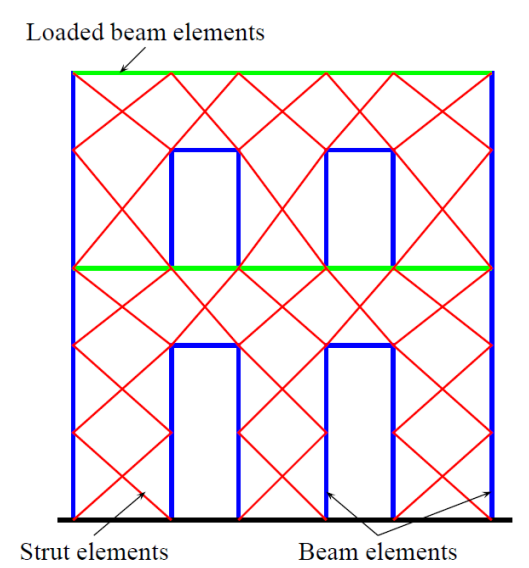

Figure 3. Building scheme.

In order to define appropriate ground motions to the non-linear dynamical analysis, a selection of 168 recorded accelerograms from the Pacific Earthquake Engineering Research Center (PEER) database have been used as inputs to the model. The events range between 5.2 and 7.6 in magnitude and the recordings have site-to-source distances from 15 to $50 \mathrm{~km}$ and dense-to-firm conditions (i.e., $360 \mathrm{~m} / \mathrm{s}<V_{s 30}<800$ $\mathrm{m} / \mathrm{s}$, where $V_{s 30}$ is the average shear wave velocity in the upper $30 \mathrm{~m}$ ).

The information carried by each single earthquake signal has been synthetized by the 13 IM parameters reported in Table 1 . These are the $M(M=$ 13) model inputs considered (Y). 
Table 1. IM earthquake parameters (model inputs: $\boldsymbol{Y}=y_{1}, y_{2}$, $\left.\ldots, y_{M=13}\right)$.

\begin{tabular}{|c|c|c|}
\hline$y_{1}$ & $I_{\text {Arias }}$ & Arias intensity \\
\hline$y_{2}$ & $P S A\left(T_{s t r}\right)$ & $\begin{array}{l}\text { spectral acceleration at the first-mode period } \\
\text { of the structure }\end{array}$ \\
\hline$y_{3}$ & $T_{m}$ & mean period \\
\hline$y_{4}$ & $a_{\max }$ & maximal outcropping acceleration \\
\hline$y_{5}$ & $T_{p}$ & predominant period \\
\hline$y_{6}$ & $D_{595}$ & significant duration \\
\hline$y_{7}$ & $I_{r m s}$ & root-mean-square intensity \\
\hline$y_{8}$ & $T_{V / A}$ & period of equivalent harmonic wave \\
\hline$y_{9}$ & $p g v$ & peak ground velocity \\
\hline$y_{10}$ & $I D$ & Cosenza and Manfredi dimensionless index \\
\hline$y_{11}$ & $S I$ & spectral intensity \\
\hline$y_{12}$ & $P S V$ & $\begin{array}{l}\text { spectral velocity at the first-mode period of } \\
\text { the structure }\end{array}$ \\
\hline$y_{13}$ & $S d$ & $\begin{array}{l}\text { spectral displacement at the first-mode period } \\
\text { of the structure }\end{array}$ \\
\hline
\end{tabular}

In the following, we refer to the model inputs by their numbers (i.e., $y_{1}, y_{2}, y_{3} \ldots$ ) instead of their name $\left(I_{\text {Arias }}, P S A\left(T_{s t r}\right), T_{m}, \ldots\right)$ for brevity.

\subsection{Results}

In order to train, validate and test the ANN, the 168 data are partitioned as follows: $70 \%$ to the training set (i.e., 118 data), $15 \%$ to the validation set (i.e., 25 data) and $15 \%$ to the test set (i.e., 25 data). This data partition is kept for all the following analyses.

As a first analysis, the ANN has been trained, validated and tested considering all the $M$ inputs. The number of nodes in the hidden layer has been chosen equal to $6(h=6)$ since (i) the number of parameters that have to be estimated in this case is 91 that is lower than the total number of training data (118), and (ii) the network thereby obtained provides the lowest Root Mean Square Error (RMSE) on the validation data set.

Table 2 shows the RMSE on the training, validation and test data sets by performing a $k$-fold cross validation (Arlot \& Celisse 2010) of the ANN considering $M=13$ inputs. The $k$-fold cross validation consists in partitioning in $k$ folds the data set obtaining by joining the training and validation data sets and considering $k-1$ folds for the training and the remaining one for validation. The process is repeated $k$ times: at each repetition the performance of the ANN is calculated. Finally, the $k$ results from the folds are averaged to produce a single RMSE estimation.

Then, the procedure of Section 4 has been applied to optimally reduce the number of features. The inputs selected are $m=6$ and they correspond to the inputs $y_{1}, y_{2}, y_{3}, y_{5}, y_{9}$ and $y_{11}$ (i.e., $I_{\text {Arias }}, \operatorname{PSA}\left(T_{\text {str }}\right)$, $T_{m}, T_{p}, p g v$ and $S I$, respectively). The number of hidden nodes $h$ identified by the algorithm is 5 . The
RMSE evaluated on the test turns out to be equal to 0.101 .

Table 3 shows the RMSE of the training, validation and test data sets by performing a $k$-fold crossvalidation of the ANN considering the $m=6$ inputs selected by the wrapper approach.

Table 2. RMSE of the cross validation of the ANN considering $M=13$ inputs.

\begin{tabular}{lc}
\hline & $\mathrm{RMSE}$ \\
Inputs & $y_{1}, y_{2}, \ldots, y_{13}$ \\
\hline Training & 0.128 \\
Validation & 0.154 \\
Test & 0.156 \\
\hline
\end{tabular}

Table 3. RMSE of the cross validation of the ANN considering $m=6$ inputs selected by the wrapper approach.

\begin{tabular}{lc}
\hline & $\mathrm{RMSE}$ \\
Inputs & $y_{1}, y_{2}, y_{3}, y_{5}, y_{9}, y_{11}$ \\
\hline Training & 0.129 \\
Validation & 0.141 \\
Test & 0.147 \\
\hline
\end{tabular}

Notice that the GA-searching scheme explored here screens unimportant features on the basis of one single objective function that is the minimization of the RMSE on the test data set. Thus, this single objective optimization does not guarantee that the number of features selected is the minimum possible.

Looking at the correlation among the $m=6$ features selected, it can be noticed that input $y_{9}$ and input $y_{11}$ are strongly correlated (i.e., correlation coefficient equal to 0.97). This may suggest that one of the two can be unnecessary and therefore neglected. Table 4 shows the comparison between the RMSE values by performing a $k$-fold cross-validation of the ANN considering $m=5$ inputs, that are inputs $y_{1}, y_{2}$, $y_{3}, y_{5}, y_{9}$ (first column) and inputs $y_{1}, y_{2}, y_{3}, y_{5}, y_{11}$ (second column). It can be noticed that the RMSE on the test removing the input $y_{9}$ is 0.140 that is lower than the RMSE on the test removing the input $y_{11}$ (RMSE = 0.162). This value is also lower than the RMSE on the test considering the $m=6$ features selected by the wrapper approach (see Table 3 ).

Thus, since the ANN provides a better performance considering the inputs $y_{1}, y_{2}, y_{3}, y_{5}$ and $y_{11}$, we consider them as the optimal feature subset for the following analyses.

Table 4. RMSE of the cross validation of the ANN considering as inputs $y_{1}, y_{2}, y_{3}, y_{5}, y_{9}$ (first column) and $y_{1}, y_{2}, y_{3}, y_{5}, \mathrm{y}_{11}$ (second column)

\begin{tabular}{lcc}
\hline & RMSE & RMSE \\
Inputs & $y_{1}, y_{2}, y_{3}, y_{5}, y_{9}$ & $y_{1}, y_{2}, y_{3}, y_{5}, y_{11}$ \\
\hline Training & 0.133 & 0.136 \\
Validation & 0.144 & 0.150 \\
Test & 0.162 & 0.140 \\
\hline
\end{tabular}


Figure 4 shows the linear regression between the network outputs $\delta_{\mathrm{ANN}}$ (on the vertical axis) and the FEM outputs $\delta_{\text {FEM }}$, i.e., the targets (on the horizontal axis), with respect to the training (Figure 4 a), validation (Figure $4 \mathrm{~b}$ ), test (Figure $4 \mathrm{c}$ ) and the entire data set (Figure $4 \mathrm{~d}$ ), considering the optimal feature subset previously identified. For a perfect fit, the data should fall along a 1:1 line, where the network outputs are equal to the targets. In this case, the fit is good for all data sets, since the correlation coefficients, $R$, are all higher than 0.94 .
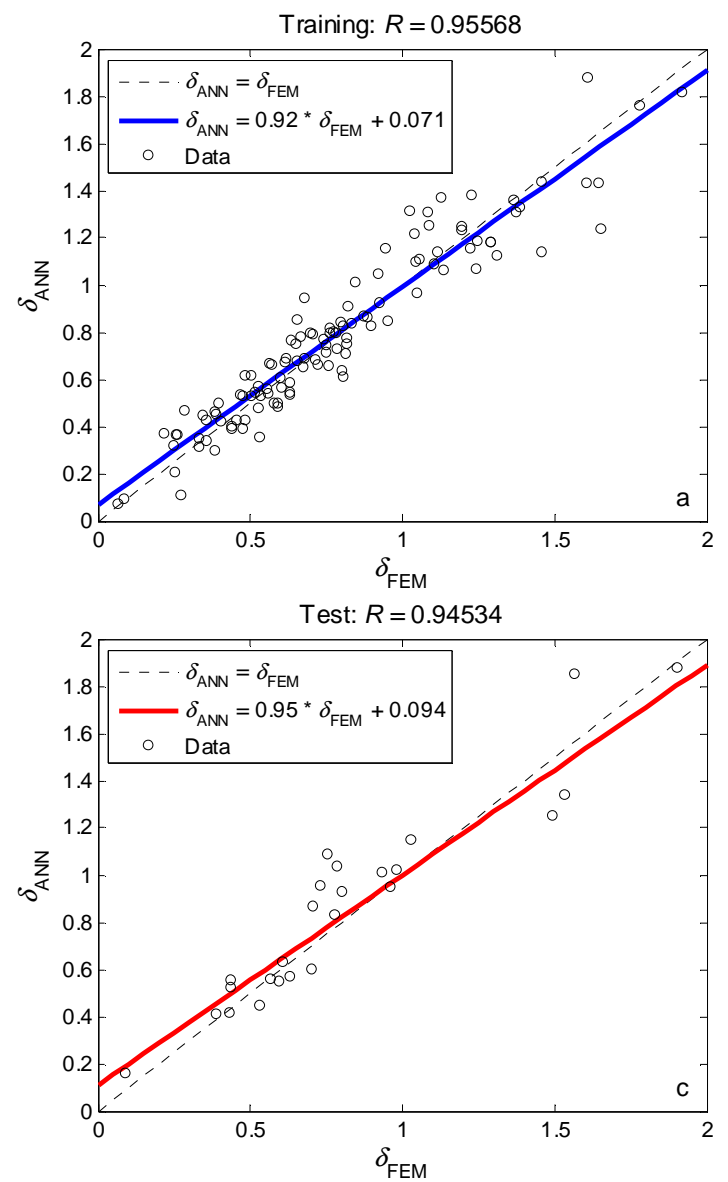

Figure 4. Linear regression between the network outputs $\left(\delta_{\mathrm{ANN}}\right.$, on the vertical axis) and the FEM outputs $\left(\delta_{\mathrm{FEM}}\right.$, on the horizontal axis).
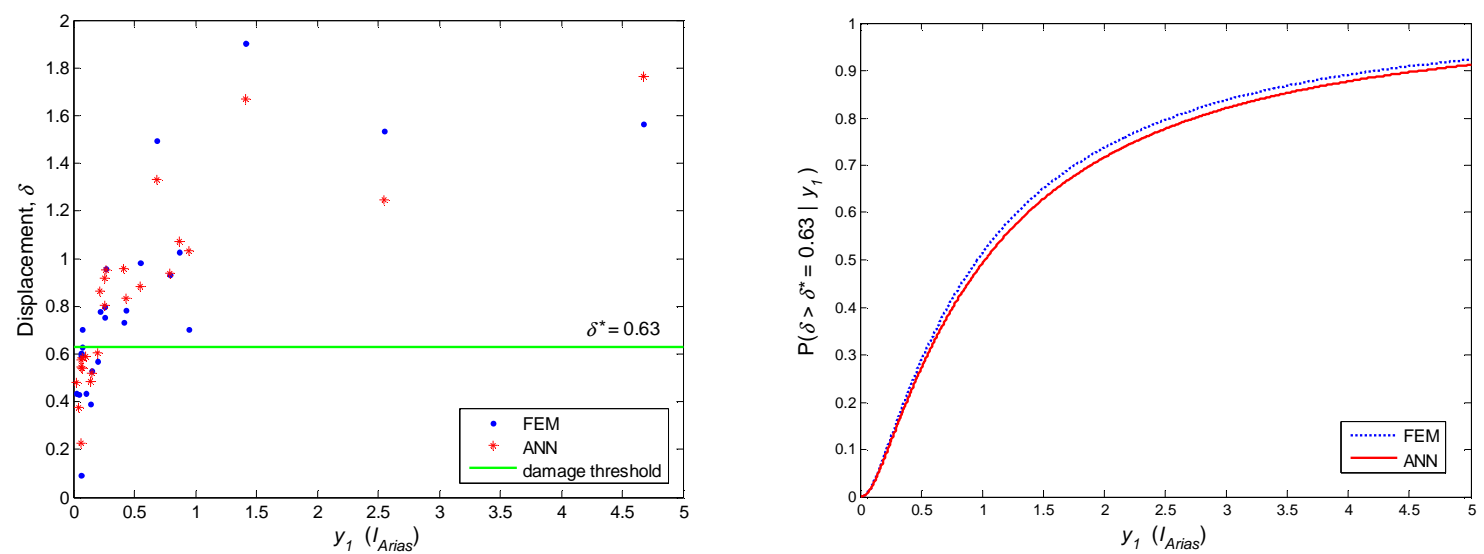

Figure 5. On the left, plot of the $y_{1}$ and the outputs obtained by the Finite Element Model (FEM), dots, and the Artificial Neural Network (ANN), stars. The horizontal line represents the damage threshold considered to build the fragility curves illustrated on the right for the FEM (dotted line) and the ANN (solid line).

Finally, the network and the FEM outputs (i.e., the displacement $\delta$ ) of the test data set have been plotted in Figure 5, left, with respect to the model input $1\left(y_{1}=I_{\text {Arias }}\right)$. A damage threshold of $0.63\left(\delta^{*}\right.$ $=0.63$ ) has been considered to build the corresponding fragility curves illustrated in Figure 5, right.

It can be noticed that the fragility curves are very similar: the parameters $\alpha$ and $\beta$ of the fragility curve estimated by the ANN are 1.0223 and 1.1749 , respectively; they are close to the ones obtained by the $\operatorname{FEM}(\alpha=0.9593$ and $\beta=1.1593)$.
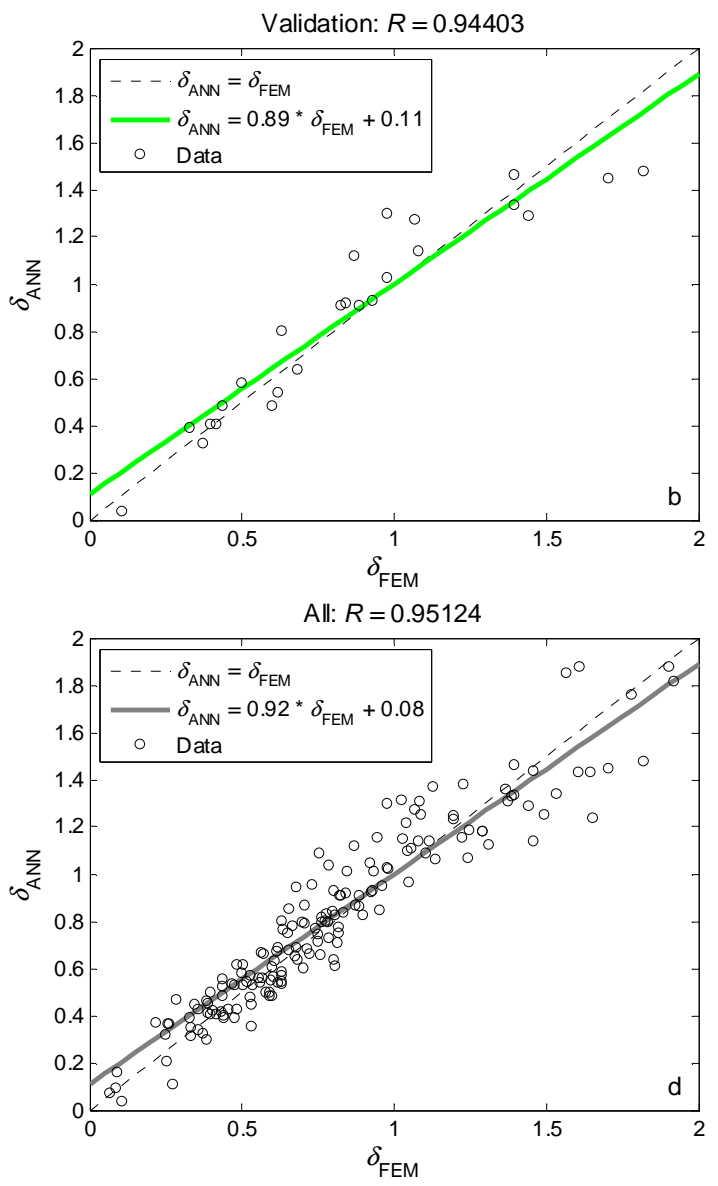


\section{CONCLUSIONS}

In this work, we have focused on the structural (failure) behavior of a masonry structure under the occurrence of a seismic event. In particular, we have replaced a Finite Element Model (FEM), typically adopted in this context to simulate the structural response of a system, by a fast-running regression model, i.e., the Artificial Neural Network (ANN) to reduce the computational burden associated to the analysis.

The following analyses have been performed:

(a) Training, validation and test of an ANN using all the $M$ inputs $(M=13)$ available from the FEM simulations;

(b) Genetic Algorithm (GA)-based wrapper feature selection to select the subset of important inputs that maximize the ANN performance;

(c) Training, validation and test of the ANN built on the optimal feature subset identified at the previous step (b) and comparison between the fragility curves determined by the FEM and the ANN outputs, respectively.

In general, with respect to the case study considered, the ANN has shown a good capability of approximating the FEM output. It can be seen that the interpolation capabilities of the metamodel increase by reducing the number of inputs (analysis (b)), since a slightly lower Root Mean Square Error (RMSE) on the test data set is obtained. In particular, at the end of the analysis, five inputs have been selected, which are: $I_{\text {Arias }}, P S A\left(T_{s t r}\right), T_{m}, T_{p}$, and $S I$. Traditionally, the Intensity Measures (IM) taken into account for this kind of analyses are the peak ground acceleration or the spectral response value at a given period. The results obtained point out that so as to improve the model prediction of the ANN and to provide more information for the output evaluation, a vector-valued IM should be considered.

The capability of the ANN to provide results similar to the FEM has been confirmed by the comparison between the fragility curves obtained by the two methods on the test data set (analysis (c)).

Future work will address the KAshiwazakiKariwa Research Initiative for Seismic Margin Assessment (KARISMA) benchmark (IAEA 2014) that refers to the real case of a NPP (unit 7 of the site Kashiwazaki Kariwa) affected, in 2007, by a strong earthquake (beyond the design criteria) and for which a rich data set (measurements and analytical methods) is available.

\section{ACKNOWLEDGEMENT}

This work, within the SINAPS@ project, benefited from French state funding managed by the National Research Agency under program RNSR Future In- vestments bearing reference No. ANR-11-RSNR0022-04. The research reported in this paper has been supported in part by the SEISM Paris Saclay Research Institute.

\section{REFERENCES}

Arlot, S., and Celisse, A. 2010. A survey of cross-validation procedures for model selection. Statistic Survey, 4, 40-79.

Aubry D., Chouvet D., Modaressi A., and Modaressi H. 1986. GEFDYN: Logiciel d'Analyse de Comportement Mécanique des Sols par Eléments Finis avec Prise en Compte du Couplage Sol-Eau-Air. Manuel Scientifique. Ecole Centrale Paris, LMSS-Mat.

Bishop, C. M. 1995. Neural Networks for pattern recognition. Oxford University Press.

Buckner, M., Gribok, A., Urmanov, A., and Hines, J. W. 2002. Application of Generalized Ridge Regression for Nuclear Power Plant Sensor Calibration Monitoring. 5th International Conference on Fuzzy Logic and Intelligent Technologies in Nuclear Science (FLINS), Gent, Belgium.

Cybenko, G. 1989. Approximation by superpositions of a sigmoidal function. Mathematics of Control Signals Systems, 2, 303-314.

Emmanouilidis, C., Hunter, A., MacIntyre, J., and Cox, C. 1999. Selecting features in neurofuzzy modelling by multiobjective genetic algorithms. In Proc. 9th Int. Conf. Artificial Neural Networks (ICANN '99) Edinburgh, UK.

EPRI. 2003. Seismic Probabilistic Risk Assessment Implementation Guide. TR-1002989, Palo Alto, CA.

Hemez, F. M., and Atamturktur, S. 2011. The dangers of sparse sampling for the quantification of margin and uncertainty. Reliability Engineering \& System Safety, 96(9), 1220-1231.

IAEA. 2014. Review of Seismic Evaluation Methodologies for Nuclear Power Plants Based on a Benchmark Exercise. IAEA-TECDOC-1722.

Lopez-Caballero, F., Modaressi-Farahmand-Razavi, A., and Tabbakhha, M. 2011. Effects of soil foundation on the performance of masonry buildings. Proceedings of the 8th International Conference on Structural Dynamics, EURODYN 2011, Leuven, Belgium

Na, M. 1997. Failure detection using a fuzzy neural network with an automatic input selection algorithm. Intelligent Hybrid Systems. Fuzzy Logic, Neural Network, and Genetic Algorithms, Ruan, D., ed., New York: Springer.

NASA. 2010. Risk-Informed Decision Making Handbook. NASA/SP-2010-576 - Version 1.0.

Rumelhart, D. E., Hinton, G. E., and Williams, R. J. 1986. Learning internal representations by error backpropagation. Parallel distributed processing: exploration in the microstructure of cognition (vol. 1), Rumelhart, D. E. and McClelland, J. L., eds., Cambridge (MA): MIT Press.

Saez, E., Lopez-Caballero, F., and Modaressi-FarahmandRazavi, A. 2011. Effect of the inelastic dynamic soilstructure interaction on the seismic vulnerability assessment. Structural Safety, 33(1), 51-63

USNRC. 2009. Guidance on the Treatment of Uncertainties Associated with PRAs in Risk-Informed Decision Making. NUREG-1855. US Nuclear Regulatory Commission, Washington, DC.

Verikas, A., and Bacauskiene, M. 2002. Feature selection with neural networks. Pattern Recognition Letters, 23(11), 13231335 .

Zio, E., Baraldi, P., and Pedroni, N. 2006. Selecting features for nuclear transients classification by means of genetic algorithms. IEEE Transactions on Nuclear Science, 53(3), 1479-1493. 